\title{
Gender-related barriers to service access and uptake in nutrition programmes identified during coverage assessments
}

\author{
Zuza Inés ${ }^{1}$, Perez Beatriz ${ }^{1}$, Ituero Clara², Das Sanjay Kumar ${ }^{3}$, Woodhead Sophie ${ }^{1}$, Rogers Eleanor ${ }^{1}$ \\ and Alvarez Jose Luis ${ }^{1}$ \\ ${ }^{1}$ Coverage Monitoring Network (CMN) at Action Against Hunger UK, First Floor, Rear Premises, 161-163 \\ Greenwich High Road, London, SE10 8JA, UK \\ ${ }^{2}$ Action Against Hunger Canada, 720 Bathurst St, Suite 500, Toronto, ON, Canada \\ ${ }^{3}$ Save the Children, 1 St John's Lane, London EC1M 4AR, UK
}

\section{Author contributions}

JLA design and developed the protocol. IZ, DSK and SW conducted the surveys work, extracted data and implemented the analyses. JLA, IZ and CI verified and conducted further analyses and drafted the manuscript with input from ER and SW. All authors provided input and approved the manuscript.

\section{Acknowledgements}

The authors would like to thank all partners and colleagues who contributed to this process including colleagues at ACF and non-governmental organisations of the Coverage Monitoring Network. The authors would also like to thank Saul Guerrero for his guidance.

\section{Conflict of Interest Statement}

The authors declare there are no conflicts of interest 


\section{Abstract}

\section{Background}

Worldwide, in 2013, approximately 17 million children under five years of age suffered from severe acute malnutrition (SAM). Although the many frequent barriers to service access in Community Management of Acute Malnutrition (CMAM) programmes have already been identified, there has been no analysis of the relationship between gender-related barriers and access to treatment.

\section{Approach}

The main objective is to review the key gender barriers to service access and uptake of treatment in CMAM programmes based on evidence gathered from coverage assessments carried out in Sub-Saharan Africa and Asia. A retrospective review of twenty-five coverage assessments from 2013 was undertaken.

\section{Main findings}

Gender-related barriers were detected in 24 of the 25 coverage assessments. The main barriers detected were related to traditional gender roles: Women busy with duties (27.8\%) followed by men control household expenditures and decision-making in the family (16.7\%) and women sick (15.3\%).

\section{Principal conclusions}

- Gender-related barriers are present in the majority of the nutrition programs, so having universal coverage as a goal, gender-related considerations must be an integral component of coverage assessment

- Initiatives contributing to gender equality would also contribute to overcoming coverage barriers in CMAM programmes

- $\quad$ The traditional use of the CMAM model considered gender only with regards to the impact that the intervention could have on gender relations. Gender has also been shown to be a key factor contributing to poor nutrition.

- Further research and assessments should include Gender Based Violence as a single barrier, or as a component of other barriers

- Coverage assessments need to include a gender approach - with tools to describe and identify gender related barriers. 


\section{Introduction}

Globally approximately 52 million children suffer from acute malnutrition (AM) at any one time (1). Over $90 \%$ of these children are in South and Southeast Asia and Sub-Saharan Africa, of whom 17 million are severely acutely malnourished (SAM). The United Nations estimates that approximately one million children under the age of five die every year from SAM. (1)

There is strong evidence that the management of SAM is a cost-effective way to reduce child mortality (2). However, for this nutrition-specific intervention to make a substantial difference, treatment coverage needs to be improved. Currently only $7-13 \%$ of the identified needs are being met, leaving over $85 \%$ of cases untreated (3). This low coverage is largely the result of limited availability and accessibility of treatment. Multiple factors impact treatment accessibility and it is only through assessing programme coverage and conducting a barrier analysis that the factors hindering access can be identified.

Community-based Management of Acute Malnutrition (CMAM) is an approach for treating acute malnutrition in young children. It consists of an outpatient model in which treatment relies on ready to use therapeutic food (RUTF) and weekly visits to monitor growth together with an inpatient model for initial treatment of complicated cases. $(4,5)$ The CMAM model includes an additional community component that delivers mobilisation activities, screening, follow-up, counselling and education. CMAM thus facilitates early detection of malnutrition, allowing children to receive treatment suited to their nutritional and medical needs. Most of these children can be rehabilitated at home, both severe and moderate cases, with a small proportion of them requiring long in-patient care.

The impact of CMAM programs is measured by performance indicators such as cure rates, but also by coverage measurements to understand the total number of children needing treatment in a population. Initially, coverage assessments were difficult to implement but the uptake of coverage measurement has increased since the availability of new, easy to use methodologies. Understanding the barriers to service access and uptake in CMAM programmes is a key element of research, investigating how to achieve universal coverage. The most common barriers to accessing SAM treatment services relate to distance, high opportunity costs, knowledge of services, and knowledge of malnutrition. Context-specific interventions are required to address and tackle the barriers preventing the community's ability to access treatment, advise and care (6). Although the most frequent barriers for accessing CMAM have been identified (7), no research has been carried out to understand the relationship between gender relations and access to treatment.

It is accepted that gender inequality can be both a cause and an effect of hunger and malnutrition since gender and nutrition are both part of the vicious cycle of poverty. (8) On the other hand, high levels of gender equality (more equal access to assets raises agricultural output and increases investment in child education) raises household food security and accelerates child growth and development. (9) Additionally, children's health services, nutrition education for mothers and women's empowerment interventions have been shown to reduce child stunting especially when applied during the first 1000 days. (8) Targeting women in nutrition interventions is often seen as the only need in the gender agenda but often women are already overburdened and therefore giving them additional tasks should be done only with careful analysis of any opportunity or other costs involved. Nutrition actually can and should offer opportunities to address sensitive issues regarding gender roles, through nutrition education, school-based and youth programmes, as well as agriculture extension. (8)

Gender differences influence child survival, health and nutrition and there is a well-known association between mothers' education and child mortality (10). Furthermore, women's ability to access and control the use of resources for their own health and well-being, division of labour and socio-cultural values are factors that have been proven to impact significantly on their children's survival, health and nutrition (11). 
The main objective of this article is to outline the key gender-related barriers ${ }^{1}$ to service access and uptake in CMAM programmes. Through detailing the main gender-related barriers that have been found through several coverage assessments, this analysis aims to contribute to the awareness and the understanding of the importance of gender sensitive programming in CMAM practices. It is also intended to provide practical guidance to programme coordinators and implementers, at national and sub-national levels, as a tool to overcome the gender-related barriers to CMAM service access. Additionally it will contribute to the global evidence base available to policy makers on the influence and role of gender, highlighting the importance of working on initiatives contributing to gender equality.

\section{Materials and methods}

Semi Quantitative Evaluation of Access and Coverage (SQUEAC) (12) methodology assessments conducted by the Coverage Monitoring Network (CMN) in Sub-Saharan Africa and Asia included 105 assessments in 2013. Twenty-five of these were purposively selected for the present study.

\section{The SQUEAC methodology}

SQUEAC (12) is a set of tools used to assess access and coverage, two essential determinants of impact for CMAM programming (13). The methodology includes two distinctive stages. First, through a thorough investigation, it collects and analyses both quantitative data from routine programme monitoring activities as well as qualitative data collected from the field. Qualitative data are collected to investigate programme operations, to unravel the opinions and experiences of multiple actors involved in CMAM and to identify any potential barriers to service access and uptake. Key informants directly and indirectly involved in the CMAM programme are interviewed, providing information on how they interact with the programme which reveals boosters and barriers to service access. Informants include: women and men from the community, programme staff, local authorities, community health workers, mothers or carers of SAM children, informal carers (traditional healers and traditional birth attendants) and any organisations supporting service delivery.

The second stage is a small area survey where enumerators find SAM cases and classify them as receiving or not receiving treatment. If a child has SAM and is not receiving treatment, a questionnaire is used to obtain basic information on why not and interviews are followed to complete the research in barriers. The information from the quantitative and qualitative investigation and the small-area survey are combined using a Bayesian approach to estimate a coverage rate and identify the key boosters and barriers to access to CMAM services. Finally an action plan is developed and implemented to overcome these barriers.

Access barriers can therefore be identified during the first, "investigation" stage where they can be quantified and weighted (in regards to the relative importance that they have on coverage) or during the second, "survey" stage where they can only be listed due to the more closed nature of the questionnaires used. In this paper we compare barriers from those two stages and identify those which are gender related.

A database was designed after the retrospective review of the coverage surveys. The main parameters analysed were as follows: administrative (ID, date of the assessment and evaluator); geographical (country and location of the assessment); number of total negative factors or barriers influencing coverage found in the investigation, and survey stages and their relative weight compared to other barriers; and identification of those barriers that are gender related as well as the sources of information for each barrier

\section{Gender-related barriers to service access and uptake in CMAM programmes}

Barriers in the SQUEAC methodology are factors which limit initial or continuous access to CMAM services and thus limit programme coverage. A previous analysis found that the five most frequently barriers were 1)

${ }^{1}$ Gender-Related Barriers do not refer here neither to the sex of the child with SAM, nor to Gender Based Violence. 
lack of knowledge of malnutrition 2) lack of knowledge of the CMAM programme 3) high opportunity costs, 4) distance to site and 5) previous rejection by services providers that discourages mothers to come back (7).

Although gender was not included in this list, many of these barriers are rooted in local social norms, and therefore in gender, especially in regards to decision making, power and control over resources and gender inequality in the community.

\section{Results}

\section{Coverage assessment characteristics}

Of the 25 SQUEAC assessments included in the analysis, 22 were from Sub-Saharan Africa and 3 from Asia, amounting to 19 countries: Angola, Burkina Faso, Cameroon, Chad, Ethiopia, Ivory Coast, Kenya, Mali, Mauritania, Niger, Nigeria, Senegal, Sierra Leone, Somali, South Sudan, Sudan, Pakistan, Philippines and Yemen. One assessment from Nigeria was excluded because it did not have all of the required data as it was an SQUEAC investigation within a Simplified Lot Quality Assurance Sampling Evaluation of Access and Coverage. Thus 24 assessments were included in the final analysis.

The weight attributed to each barrier, which reflects the relative importance that they have on coverage, could be obtained from 19 assessments.

\section{Classification of gender-related barriers}

In the SQUEAC methodology, barriers found to be caused by gender relations are classified as 'gender-related barriers'. Gender-related barriers were analysed and classified into two categories. These categories were not predefined - they emerged once the gender-related barriers were more closely examined. These categories were:

1. Gender Roles and Responsibilities:

- $\quad$ Related to the workload and traditional roles of men and women in each context. In all of the countries assessed, women are the principal carers of children. They are also responsible for taking malnourished children to health facilities, while men are providers and protectors of the family.

- $\quad$ Stigma of having a malnourished child. Women or family members can be ashamed of having a malnourished child, resulting in the child not accessing treatment. If a child is malnourished, a woman can perceive that she failed in her role as care-giver. Additionally, the stigma can be related to the perception of malnutrition as a disease of poverty or to frequent pregnancies.

2. Gender-based Discrimination:

This is related to the imbalance of gender relations and how this discrimination plays out in their daily lives. It includes:

Decision making. In each community and culture the members of a family have different levels of decision-making power e.g. needs of health care and management of household expenses. There is often an imbalance in decision-making power. Normally men take decisions, though other members of the community, such as mothers-in-law, can also hold considerable power.

- $\quad$ Level of education and socio-cultural practices. The level of education is generally low in the countries included in the analysis and usually differ between men and women. Women traditionally have less access to education and have lower literacy rates than men, often resulting in less access to information. This can perpetuate unhealthy beliefs and practices. As the level of education differs between males and females, there are often less female community health workers which can affect the communication of health messages and their uptake. 
Early Marriage and motherhood affects women's level of education and development. If a woman is married and pregnant at a young age, she is likely to be more vulnerable and dependent on an influential family member, potentially leading to the continuation of traditional practices.

\section{Gender-related barriers}

Gender-related barriers to service access and uptake in CMAM programmes were detected in 23 of the 24 assessments and are described in Table 1. In total, 69 gender-related barriers were found in the 23 assessments, with a mean of 2.8 (SD: 1.3) barriers per evaluation.

- 58 (84\%) of the gender-related barriers were identified during the investigation stages of the SQUEAC assessments and 47 of those barriers were weighted

- 11 (16\%) gender-related barriers were identified during the field survey stages.

Table 1 Gender-related barriers to service access and uptake in CMAM programmes detected in coverage assessments developed by the CMN project in 2013

\begin{tabular}{|c|c|c|c|c|c|}
\hline & $\begin{array}{l}\text { Gender-related barriers to service access and uptake } \\
\text { in CMAM programmes }\end{array}$ & $\begin{array}{c}\text { No. } \\
\text { identified } \\
\text { during } \\
\text { investig. } \\
\text { stages }\end{array}$ & $\begin{array}{c}\text { No. } \\
\text { identified } \\
\text { during } \\
\text { field } \\
\text { survey } \\
\text { stages }\end{array}$ & $\begin{array}{c}\text { Total No. } \\
\text { of } \\
\text { gender- } \\
\text { related } \\
\text { barriers }\end{array}$ & $\begin{array}{l}\% \text { of } \\
\text { total }\end{array}$ \\
\hline \multirow{6}{*}{$\begin{array}{l}\text { Gender Roles } \\
\text { and } \\
\text { Responsibilities }\end{array}$} & $\begin{array}{l}\text { Carer busy with duties (household, agricultural, animals, } \\
\text { water and with other children) }\end{array}$ & 17 & 4 & 21 & $30 \%$ \\
\hline & Carer sick & 5 & 5 & 10 & $15 \%$ \\
\hline & Father feels ashamed because he did not support the family & 3 & 0 & 3 & $4 \%$ \\
\hline & Mother pregnant & 0 & 1 & 1 & $1 \%$ \\
\hline & Mother with malnourished child is perceived to be negligent & 1 & 0 & 1 & $1 \%$ \\
\hline & Carer busy in social events & 1 & 0 & 1 & $1 \%$ \\
\hline \multirow[t]{8}{*}{$\begin{array}{l}\text { Gender-based } \\
\text { Discrimination }\end{array}$} & $\begin{array}{l}\text { Men control household expenditures and decision-making } \\
\text { in the family: husband refuses to bring the child to the } \\
\text { health facility }\end{array}$ & 10 & 1 & 11 & $16 \%$ \\
\hline & $\begin{array}{l}\text { Frequent pregnancies/ sexual intercourse with lactating } \\
\text { women and stigma of being frivolous that makes them feel } \\
\text { ashamed to go the health services }\end{array}$ & 10 & 0 & 10 & $15 \%$ \\
\hline & $\begin{array}{l}\text { Low level of woman's literacy prevents uptake of messages } \\
\text { for health and nutrition education }\end{array}$ & 4 & 0 & 4 & $6 \%$ \\
\hline & $\begin{array}{l}\text { Mother-in-law/ family refuse to bring the child to the health } \\
\text { facility }\end{array}$ & 3 & 0 & 3 & $4 \%$ \\
\hline & Mother too young / early motherhood / early marriage & 2 & 0 & 1 & $3 \%$ \\
\hline & $\begin{array}{l}\text { Cultural perceptions and practices surrounding pregnancy } \\
\text { and childbirth }\end{array}$ & 1 & 0 & 1 & $1 \%$ \\
\hline & Few community health workers are women & 1 & 0 & 1 & $1 \%$ \\
\hline & Total & 58 & 11 & 69 & \\
\hline
\end{tabular}

About $75 \%$ of cases fell into the four most common categories. Sub-Saharan Africa and Asia had similar distributions of barriers.

37 (54\%) barriers belonged to the "gender roles and responsibilities" categories and 32 (46\%) relate to "gender-based discrimination." 


\section{Relation of the gender-related barriers with the overall barriers of the}

\section{assessment}

When looking at the relation of the gender-related barriers to the overall barriers found during the investigation stage of the assessments, in total 416 barriers were found, with a mean of 17.3 (SD: 7.6) barriers per evaluation. From those, 47 are gender-related barriers (or a combination of multiple gender-related barriers); therefore, $11 \%$ of the barriers in the quantitative assessments were gender related.

The weight assigned to each barrier during the SQEAC investigation was obtained for 333 barriers (39 of them gender-related barriers) from 19 assessments. The total weighted scores of those 333 barriers was 953, compared with the sum of the scores of the gender barriers which is 102 . This means that $11 \%$ of the relative importance of the barriers in the assessment was gender related. The gender related barrier with the highest score when adding all of the weights assigned in each assessment was "carer busy with duties (household, agricultural, animals, water and with other children)" which represents 17 out of 58 (29\%) gender barriers or 17 out of 416 (4\%) from the total of the barriers.

\section{Key informants on gender-related barriers}

The types of key informants that mentioned gender-related barriers during interviews most frequently are shown in Figure 1.

Figure 1: Key informants - that provided information on gender-related barriers to service access and uptake in CMAM programmes - in selected coverage assessments developed by the CMN project in 2013

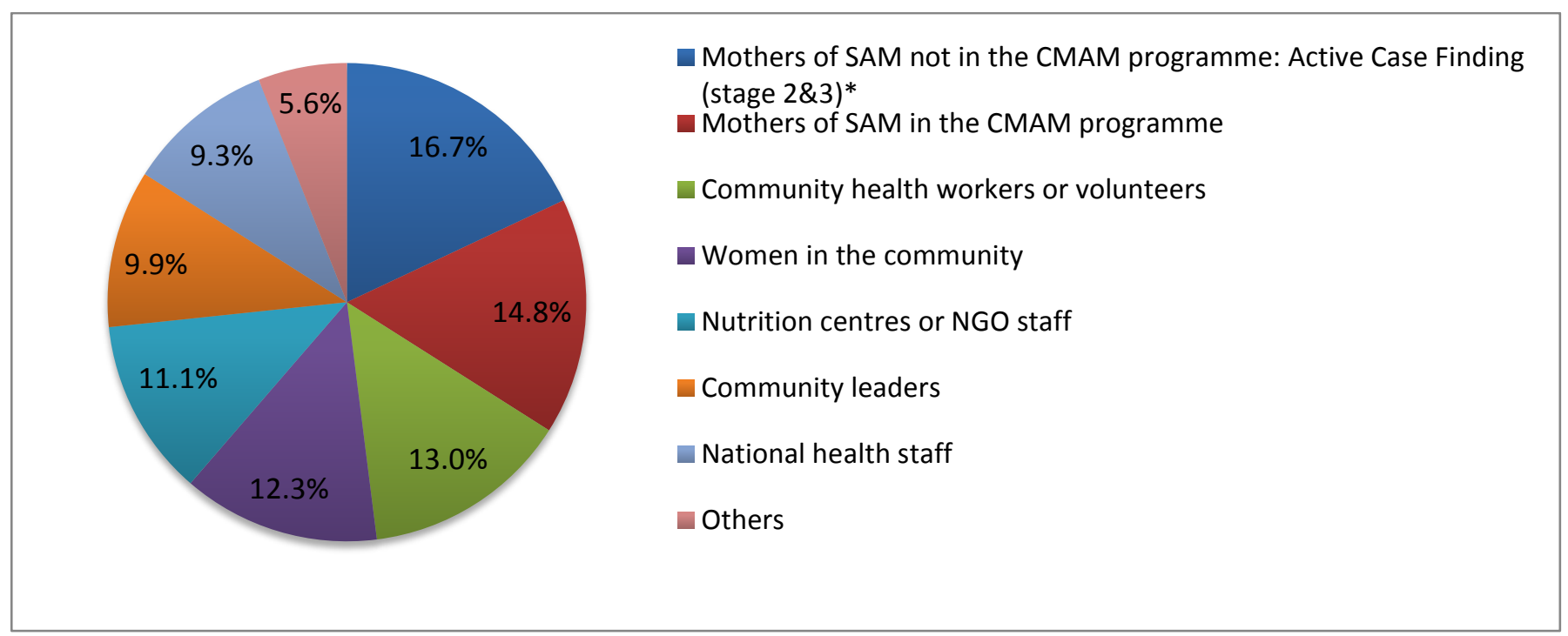

* Mothers or caretakers of severe acute malnourished children detected during the active and adaptive case finding.

Mothers or caretakers of SAM children are the most common informants of gender-related barriers, representing $32 \%$ of the key informants. Community health workers or volunteers and other women in the community are also an important source of gender-related information representing $13 \%$ and $12 \%$ of the key informants respectively.

In the Asian assessments, the most frequent key informants were females in the community ( $\mathrm{n}=2)$, and none of the Asian assessments included the mothers or caretakers of SAM as sources.

\section{Discussion}

Gender-related barriers were present in the majority of the programmes analysed and they appeared likely to directly influence treatment seeking behaviour and use of health services. The first and most common gender- 
related barrier "carer busy with duties", suggests that the responsibilities of female carers present a very real and challenging barrier to accessing the treatment programmes. In the same category of gender-related barriers, "carer sick" represents a significant impact on the access and uptake of CMAM services. "Men control household expenditures and decision-making in the family" was also common, and may affect the level of care access by limiting mothers or caretakers power of decision-making in accessing the treatment services.

In general, a gender perspective was not taken into account when the coverage assessments were undertaken. As gender norms are embedded as part of people's lives and they depict local norms in social relations, gender barriers are frequently missed or intertwined with political, economic, cultural and social issues in coverage assessments.

Recommendations focusing on gender-related barriers are not common in coverage assessment reports. However, if we aim for universal coverage, those barriers need to be addressed and thus a gendered approach should be systematically considered in coverage assessments. Including this, an essential component of the health seeking behaviour of many populations, will increase the likelihood that recommendations focus on overcoming these barriers and improving coverage. Some examples of how this can be achieved in coverage assessments could include to have a balanced enumerator's team of women and men during data collection, to ensure adequate understanding and documenting the differences in gender roles, activities, needs, and opportunities in a given context before undertaking an assessment, and ensuring an active and inclusive participatory approach (women, girls, boys and men of all ages from the affected community, including vulnerable groups, should be given the opportunity to actively participate). (14)

The coverage methodology does not identify gender-related barriers specifically, reducing both the amount of information that could be extracted and the potential reliability of the analysis of the data. For example, 3 assessments from South Sudan were analysed. Two of them had 4 and 5 gender barriers, and the third had none. Therefore it is possible that the third assessment was implemented without any gender perspective in mind, leading to a suspicion that these sometimes go underreported because the SQUEAC methodology does not include a specific methodology to assess gender issues. Therefore some important events may have gone unnoticed or were not recorded. In some of the assessments, different gender-related barriers were combined and grouped as one barrier, which may have influenced the weight of the overall gender-related barriers. For example barriers such as "carer sick" and "frequent pregnancies" are sometimes grouped and counted as one barrier in the initial assessments.

Gender based violence (GBV), as a single gender-related barrier was not considered as a barrier in itself. From our field experience we know that it may be masked within other general barriers such as "distance to site" because in many places security is weak, especially for women walking in the dark.

Approaches to identifying gender related barriers should avoid stereotypes and be based on results from a gender analysis. Alternatives to minimize the gender related barrier "carer busy with duties" should be sought, such us involving other members of the family or the community in the malnutrition treatment or decreasing women's workload. CMAM programs need to be designed to minimize any increase in the workload of the carer, in particular during the harvesting period. Even workshops for mothers of SAM children can increase their workload (physically and emotionally) and budget planning might include compensation for opportunity costs.

Gender inequality is the root cause of many barriers to access and uptake of CMAM programs, thus, programs contributing to gender equality would improve the coverage of these programs and though gender-related barriers represent a low percentage of the total barriers faced, further research using more appropriate tools focusing on gender should be carried out. This way, gender-related barriers and analyses of the gender components of the remaining barriers, could be better identified and measured. 


\section{Implications for practice and conclusions}

This article details the main gender-related barriers that have been found through several CMAM coverage assessments in different countries. Gender-related barriers are present in the majority of these nutrition programs, so if we aim for universal coverage, gender must be an integral component of coverage assessment. Similarly, coverage assessments need to include a gender approach - with tools to describe and identify gender related barriers. The most frequent gender barrier identified was carer busy with duties. It is also the barrier which was found to have the greatest impact on programming.

Gender sensitive programming in CMAM practices can be improved by:

- Including gender systematically in nutrition coverage assessments

- Conducting a gender analysis during the needs assessment to understand gender relations in the community before any CMAM intervention

- Using findings from gender analysis and avoiding stereotypical gender assumptions, such as targeting only women for traditionally being the care takers

- Proposing ways to overcome the gender related barrier "carer busy with duties". Either involve other member of the family/ community, take into account seasonal workloads, decrease women's workload, or compensate women for the value of the time they are expected to travel and to participate in activities

- Further research and assessments should include gender-based violence as a single barrier, or as a component of other barriers 


\section{References}

(1) UNICEF-WHO-The World Bank Joint Malnutrition Estimates. 2013 Levels \& Trends in Child Malnutrition. 2015.

(2) Bhutta ZA, Das JK, Rizvi A, Gaffey MF, Walker N, Horton S, et al. Evidence-based interventions for improvement of maternal and child nutrition: what can be done and at what cost? Lancet 2013 Aug 3;382(9890):452-77.

(3) Coverage Monitoring Network. What We Know Now: a Decade of Community-based Treatment of Severe Acute Malnutrition. Conference Report. 17-18 October, 2013. 2015.

(4) Collins S, Dent N, Binns P, Bahwere P, Sadler K, Hallam A. Management of severe acute malnutrition in children. Lancet Lond Engl. 2006;368:1992-2000

(5) Manual AF. Community-based Therapeutic Care (CTC). http://www.unicefinemergencies.com/downloads/eresource/docs/2.3\%20Nutrition/Int'l\%20Comm\% 20Based\%20Therapeutic\%20Care\%20Manual.pdf. Accessed 26 Aug 2016.

(6) Puett C, Guerrero S. Barriers to access for severe acute malnutrition treatment services in Pakistan and Ethiopia: a comparative qualitative analysis. Public Health Nutr. 2015;18:1873-82

(7) Puett C, Hauenstein S, Guerrero S. Access for All, Volume 2: What factors influence access to community-based treatment of severe acute malnutrition? 2013.

(8) FAO (2012). Gender and Nutrition: http://www.fao.org/fileadmin/user_upload/wa_workshop/docs/GenderNutrition_FAO_IssuePaper_Draft.pdf

(9) Ruth Oniang’o and Edith Mukudi, "Nutrition and Gender.” In Nutrition: A Foundation for Development, Geneva: ACC/SCN, 2002

(10) Gakidou E, Cowling K, Lozano R, Murray CJ. Increased educational attainment and its effect on child mortality in 175 countries between 1970 and 2009: a systematic analysis. Lancet 2010 Sep 18;376(9745):959-74.

(11) UNICEF, LSTM. Gender Influences on Child Survival a Narrative review. 2011 Dec.

(12) Myat M, Guevarra E, Fieschi L, Norris A, Guerrero S, Schofield L, et al. Semi-Quantitative Evaluation of Access and Coverage (SQUEAC)/Simplified Lot Quality Assurance Sampling Evaluation of Access and Coverage (SLEAC) Technical Reference. 2012. Washington, DC: FHI/360 FANTA.

(13) Mark Myatt, Saul Guerrero (2013). Why coverage is important: efficacy, effectiveness, coverage, and the impact of CMAM Interventions. Field Exchange 45, May 2013. p39. www.ennonline.net/fex/45/coverage

(14) ACF Gender policy. Increasing the impact of ACF's work through gender equality programming. Gender policy and toolkit. March 2014. 